\title{
On the differences of the partition function
}

\author{
by
}

Gert Almkvist (Lund)

Introduction. A lot has been written about the differences $\Delta^{r} p(n)$ of the partition function $p(n)$ (see Odlyzko [4] for the history of the problem). Odlyzko settled the main problem by proving that $\Delta^{r} p(n)$ alternates in sign up to a certain value, $n=n_{0}(r)$, and then it stays positive. He also proved the asymptotic formula

$$
n_{0}(r) \sim \frac{6}{\pi^{2}} r^{2} \log ^{2} r \quad \text { as } r \rightarrow \infty .
$$

The convergence to the limit is, however, very slow. E.g. for $r=75$,

$$
\frac{6}{\pi^{2}} 75^{2} \log ^{2} 75=63744
$$

while $n_{0}(75)=140372$. Even for $r=1000$,

$$
\frac{6}{\pi^{2}} 1000^{2} \log ^{2} 1000=29008508
$$

while $n_{0}(1000)$ is conjectured to be 53338056 .

Using the WKB method on the difference equation for $\Delta^{r} p(n)$, Knessl and Keller [3] obtained a remarkably exact (though complicated) equation for $n_{0}(r)$. Solving it they found the correct value of $n_{0}(r)$ within 2 units up to $r=75$.

The aim of this paper is to find a more exact formula than Odlyzko's. Using the general theory in [2] we find an exact formula for $\Delta^{r} p(n)$. Taking just the first two terms and using an asymptotic formula for Bessel functions we obtain an equation which is similar to the one of Knessl and Keller. It is easier to solve for $r$ as a function of $n=n_{0}(r)$ and we get

Theorem 1. Put

$$
x_{1}=\frac{24}{\pi^{2}}\left(n_{0}(r)-\frac{r}{2}-\frac{1}{24}\right) \quad \text { and } \quad a=\frac{\pi^{2}}{6} .
$$


Then

$$
\begin{aligned}
r= & \frac{a \sqrt{x_{1}}}{\log x_{1}}\left\{1-\frac{1}{\log ^{2} x_{1}}+\frac{25}{12} \cdot \frac{1}{\log ^{4} x_{1}}-\frac{1803}{320} \cdot \frac{1}{\log ^{6} x_{1}}+\ldots\right\} \\
& +\frac{\log 2}{\log x_{1}}-\frac{3}{\log ^{2} x_{1}}-\frac{1}{2} \cdot \frac{1}{\log ^{3} x_{1}}+\frac{10}{3} \cdot \frac{1}{\log ^{4} x_{1}}+\frac{5}{4} \cdot \frac{1}{\log ^{5} x_{1}} \\
& -\frac{2363}{320} \cdot \frac{1}{\log ^{6} x_{1}}+\frac{2}{a} \cdot \frac{1}{\sqrt{x_{1}} \log x_{1}}+\frac{a}{12} \cdot \frac{1}{\sqrt{x_{1}} \log ^{2} x_{1}} \ldots
\end{aligned}
$$

Remark. Already the equation

$$
r=\frac{a \sqrt{x_{1}}}{\log x_{1}}
$$

has a solution giving rather good estimates. Thus if $r=75$ we get $n_{0}=$ 138500 , compared to $n_{0}(75)=140372$.

It seems hard to invert the formula in Theorem 1 to get $n_{0}$ as a function $r$. Here is a modest attempt.

Theorem 2. Put $r_{1}=\frac{12}{\pi^{2}} r$. Then let

$$
t_{0}=r_{1}\left(\log r_{1}+\log \log r_{1}+\log \log \log r_{1}+\ldots\right)
$$

where the sum continues as long as the terms are positive. Put

$$
t_{1}=t_{0} \cdot \frac{\log t_{0}-1}{\frac{t_{0}}{r_{1}}-1} .
$$

Then

$$
x_{1} \approx \frac{t_{1}^{2}}{\left(1-\left(\frac{r_{1}}{2 t_{1}}\right)^{2}\right)^{2}} .
$$

EXAmPLE 1 . If $r=75$, Theorem 2 gives $n_{0} \approx 140235$ compared to the correct value $n_{0}=140372$. For $r=20$ we (accidentally) get $n_{0} \approx 5622$ instead of $n_{0}(20)=5620$.

The best approximation to $n_{0}(r)$ comes from using the first two terms in the exact formula for $\Delta^{r} p(n)$. Define

$$
L_{\nu}(x)=\sum_{n=0}^{\infty} \frac{x^{n}}{n ! \Gamma(n+\nu+1)} .
$$

Then we have

Theorem 3. Put

$$
x=\frac{\pi^{2}}{6}\left(n_{0}-\frac{r}{2}-\frac{1}{24}\right) .
$$


Then $x$ is approximately the solution to the transcendental equation

$$
L_{r+3 / 2}(x)=2^{-5 / 2}\left(12 / \pi^{2}\right)^{r} L_{3 / 2}(x / 4) .
$$

Finally, a method is outlined to find an approximate solution by using Theorem 2. Then the equation in Theorem 3 is solved by using NewtonRaphson's algorithm. This is very simple since

$$
\frac{d}{d x} L_{\nu}(x)=L_{\nu+1}(x) .
$$

Thus a direct method is obtained where no fitting is necessary (as in [3]).

Proofs. The generating function for $r$ th differences is

$$
g(x)=\sum_{n=0}^{\infty} \Delta^{r} p(n) x^{n}=\frac{(1-x)^{r}}{\prod_{\nu=1}^{\infty}\left(1-x^{\nu}\right)} .
$$

Using the terminology of [2] we set

$$
F(x)=\sum_{n=0}^{\infty} p(n) x^{n}=\prod_{\nu=1}^{\infty}\left(1-x^{\nu}\right)^{-1} .
$$

Hence

$$
S(x)=\frac{g(x)}{F(x)}=(1-x)^{r} .
$$

The "general formula" together with Rademacher's formula for $p(n)$ now gives ([2], Main Theorem)

$$
\begin{aligned}
\Delta^{r}(n)=2 \pi\left(\frac{\pi}{12}\right)^{3 / 2} \sum_{k=1}^{\infty} \sum_{(h, k)=1} \omega(h, k) e^{-2 \pi i h n / k} k^{-5 / 2} & \\
& \times S\left(e^{-(D-2 \pi i h / k)}\right) L_{3 / 2}\left(\frac{\pi^{2}}{6 k^{2}}\left(n-\frac{1}{24}\right)\right)
\end{aligned}
$$

where

$$
L_{\lambda}(x)=\sum_{\nu=0}^{\infty} \frac{x^{\nu}}{\nu ! \Gamma(\nu+\lambda+1)} .
$$

First we consider only the first two terms $(\omega(1,1)=\omega(1,2)=1)$. We get

$$
\begin{aligned}
S\left(e^{-D}\right) & =\left(1-e^{-D}\right)^{r}=2^{r} e^{-r D / 2}\left(\sinh \frac{D}{2}\right)^{r} \\
& =e^{-r D / 2} D^{r}\left(1+\frac{r}{24} D^{2}+\ldots\right)
\end{aligned}
$$


and

$$
\begin{aligned}
S\left(-e^{-D}\right) & =\left(1+e^{-D}\right)^{r}=2^{r} e^{-r D / 2}\left(\cosh \frac{D}{2}\right)^{r} \\
& =2^{r} e^{-r D / 2}\left(1+\frac{r}{8} D^{2}+\ldots\right) .
\end{aligned}
$$

It follows that

$$
\begin{aligned}
& \Delta^{r}(n)=2 \pi\left(\frac{\pi}{12}\right)^{3 / 2}\left\{e^{-r D / 2} D^{r}\left(1+\frac{r}{24} D^{2}+\ldots\right) L_{3 / 2}\left(\frac{\pi^{2}}{6}\left(n-\frac{1}{24}\right)\right)\right. \\
& \left.\quad+(-1)^{n} 2^{-5 / 2} \cdot 2^{r} e^{-r D / 2}\left(1+\frac{r}{8} D^{2}+\ldots\right) L_{3 / 2}\left(\frac{\pi^{2}}{24}\left(n-\frac{1}{24}\right)\right)+\ldots\right\} .
\end{aligned}
$$

Put $a=\pi^{2} / 6$ and $x=a(n-r / 2-1 / 24)$. Then

$$
\begin{aligned}
\Delta^{r}(n)=2 \pi & \left(\frac{\pi}{12}\right)^{3 / 2}\left\{a^{r} L_{r+3 / 2}(x)+\frac{r}{24} a^{r+2} L_{r+7 / 2}(x)+\ldots\right. \\
& \left.+(-1)^{n} 2^{r-5 / 2}\left(L_{3 / 2}(x / 4)+\frac{r a^{2}}{128} L_{7 / 2}(x / 4)+\ldots\right)+\ldots\right\} .
\end{aligned}
$$

Here we use

$$
D^{r} L_{3 / 2}(a(n-1 / 24))=a^{r} L_{r+3 / 2}(a(n-1 / 24))
$$

and

$$
e^{-r D / 2} L_{\nu}(a(n-1 / 24))=L_{\nu}(a(n-r / 2-1 / 24)) \quad \text { (Taylor's theorem) } .
$$

Let us first ignore the higher order terms. Both the first term and the absolute value of the second term increase with $x$. For small $x$ the second term dominates (due to the factor $2^{r-5 / 2}$ and $L_{r+3 / 2}(0)=1 / \Gamma(r+3 / 2)$ ). Thus $\Delta^{r}(n)$ alternates in sign until the first term dominates. This will occur since $L_{r+3 / 2}(x)$ is larger than $L_{3 / 2}(x / 4)$ for large $x$ (see the asymptotic estimates used later in the proof). The critical value $n_{0}(r)$ is obtained when the two terms are equal.

Estimate of the higher order terms. By Rademacher ([5], pp. 291292) we have

$$
p(n)=2 \pi\left(\frac{\pi}{12}\right)^{3 / 2} \sum_{k=1}^{\infty} A_{k}(n) k^{-5 / 2} L_{3 / 2}\left(\frac{\pi^{2}}{6 k^{2}}\left(n-\frac{1}{24}\right)\right)
$$

and the estimate

$$
\left|A_{k}(n)\right| \leq 2 k^{3 / 4}
$$


We estimate the $r$ th difference of the $k$ th term:

$$
\begin{aligned}
\left|\Delta^{r} f_{k}\right|= & 2 \pi\left(\frac{\pi}{12}\right)^{3 / 2} k^{-5 / 2} \\
& \times\left|\sum_{\nu=0}^{r}(-1)^{\nu}\left(\begin{array}{l}
r \\
\nu
\end{array}\right) A_{k}(n-\nu) \cdot L_{3 / 2}\left(\frac{\pi^{2}}{6 k^{2}}\left(n-\nu-\frac{1}{24}\right)\right)\right| \\
\leq & 2 \pi\left(\frac{\pi}{12}\right)^{3 / 2} k^{-5 / 2} \cdot 2 \cdot k^{3 / 4} \cdot 2^{r} L_{3 / 2}\left(\frac{\pi^{2} n}{6 k^{2}}\right)
\end{aligned}
$$

since $\sum_{\nu=0}^{r}\left(\begin{array}{l}r \\ \nu\end{array}\right)=2^{r}$. Using

$$
L_{3 / 2}(x) \leq \frac{1}{2 \sqrt{\pi}} \cdot \frac{e^{2 \sqrt{x}}}{x}
$$

and the fact that $L_{3 / 2}(x)$ increases with $x$ we get

Hence

$$
L_{3 / 2}\left(\frac{\pi^{2} n}{6 k^{2}}\right) \leq L_{3 / 2}\left(\frac{\pi^{2} n}{6 \cdot 3^{2}}\right) \leq \frac{1}{2 \sqrt{\pi}} \cdot \frac{54}{\pi^{2}} \cdot \frac{e^{2 \pi \sqrt{n} /(3 \sqrt{6})}}{n} .
$$

and

$$
\left|\Delta^{r} f_{k}\right| \leq \frac{9}{2 \sqrt{3}} 2^{r} k^{-7 / 4} \frac{e^{2 \pi \sqrt{n} /(3 \sqrt{6})}}{n}
$$

$$
\sum_{k=3}^{\infty}\left|\Delta^{r} f_{k}\right| \leq \frac{9}{2 \sqrt{3}} 2^{r} \zeta(7 / 4) \frac{e^{2 \pi \sqrt{n} /(3 \sqrt{6})}}{n} .
$$

Let us compare this with the second term

$$
\begin{aligned}
\left|\Delta^{r} f_{2}\right| & \approx 2^{r-5 / 2} L_{3 / 2}\left(\frac{\pi^{2}}{24}\left(n-\frac{r}{2}-\frac{1}{24}\right)\right) \\
& \approx \frac{24 \cdot 2^{r}}{(2 \pi)^{5 / 2}} \cdot \frac{\exp \left(\frac{\pi}{\sqrt{6}} \sqrt{n-r / 2-1 / 24}\right)}{n-r / 2-1 / 24} \approx \frac{3 \sqrt{2}}{\pi^{5 / 2}} \cdot 2^{r} \frac{e^{\pi \sqrt{n} / \sqrt{6}}}{n} .
\end{aligned}
$$

Hence

$$
\frac{\sum_{k=3}^{\infty}\left|\Delta^{r} f_{k}\right|}{\left|\Delta^{r} f_{2}\right|} \leq \pi^{5 / 2} \sqrt{3 / 8} \zeta(7 / 4) e^{-\pi \sqrt{n} /(3 \sqrt{6})} \leq 25 \cdot e^{-0.4275 \sqrt{n}} .
$$

Since we have tables of $n_{0}(r)$ up to $r=75$, we may assume that $r \geq 75$ and hence $n \geq 140000$, which implies that the right hand side is $\leq 10^{-69}$ so we can safely neglect the higher order terms.

We get the equation (to determine $n_{0}(r)$ )

$$
\begin{aligned}
a^{r}\left(L_{r+3 / 2}(x)+\frac{r a^{2}}{24} L_{r+7 / 2}(x)+\ldots\right) & \\
& =2^{r-5 / 2}\left(L_{3 / 2}(x / 4)+\frac{r a^{2}}{128} L_{7 / 2}(x / 4)+\ldots\right) .
\end{aligned}
$$


Now $L_{\gamma}(x)$ is a modified Bessel function:

$$
J_{\gamma}(z)=(z / 2)^{\gamma} L_{\gamma}\left(-z^{2} / 4\right) .
$$

Using formula 9.7.7 in Abramowitz-Stegun ([1], p. 378) we get

$$
L_{\nu}(x)=\frac{1}{\sqrt{2} \pi} \cdot \frac{2^{\nu} e^{\sqrt{\nu^{2}+4 x}}}{\left(\nu^{2}+4 x\right)^{1 / 4}\left(\nu+\sqrt{\nu^{2}+4 x}\right)^{\nu}}\left\{1+\frac{6 x-\nu^{2}}{12\left(\nu^{2}+4 x\right)^{3 / 2}}+\ldots\right\} .
$$

Furthermore, we have

$$
\begin{gathered}
L_{3 / 2}(x / 4) \approx \frac{2}{\sqrt{\pi}} \frac{e^{\sqrt{x}}}{x}\left(1-\frac{1}{\sqrt{x}}\right), \\
L_{7 / 2}(x / 4) \approx \frac{8}{\sqrt{\pi}} \frac{e^{\sqrt{x}}}{x^{2}}\left(1-\frac{6}{\sqrt{x}}+\frac{15}{x}-\frac{15}{x \sqrt{x}}\right) .
\end{gathered}
$$

Thus

and

$$
L_{\nu+2}(x) \approx \frac{4}{\nu^{2}+4 x} L_{\nu}(x) \approx \frac{1}{x} L_{\nu}(x)
$$

$$
L_{7 / 2}(x / 4) \approx \frac{4}{x} L_{3 / 2}(x)
$$

Putting $\nu=r+3 / 2$ we get the equation

$$
a^{r} L_{\nu}(x)\left(1+\frac{r a^{2}}{6\left(\nu^{2}+4 x\right)}+\ldots\right)=2^{r-5 / 2} L_{3 / 2}(x / 4)\left(1+\frac{r a^{2}}{32 x}+\ldots\right) .
$$

Neglecting the second terms in the parenthesis (when $r=75$ they are of order 0.00003 ) we get

$$
\frac{L_{r+3 / 2}(x)}{L_{3 / 2}(x / 4)}=2^{-5 / 2}\left(\frac{12}{\pi^{2}}\right)^{r},
$$

which is the equation in Theorem 1.

Substituting the asymptotic expressions for the Bessel functions we get the equation

$$
\begin{aligned}
\frac{1}{\sqrt{2 \pi}} \cdot \frac{2^{\nu} a^{r} e^{\sqrt{\nu^{2}+4 x}}}{\left(\nu^{2}+4 x\right)^{1 / 4}\left(\nu+\sqrt{\nu^{2}+4 x}\right)^{\nu}}\left\{1+\frac{6 x-\nu^{2}}{12\left(\nu^{2}+4 x\right)^{3 / 2}}\right\}\left\{1+\frac{r a^{2}}{24 x}\right\} \\
=2^{r-5 / 2} \cdot \frac{2}{\sqrt{\pi}} \cdot \frac{e^{\sqrt{x}}}{x}\left(1-\frac{1}{\sqrt{x}}\right)\left(1+\frac{r a^{2}}{32 x}\right) .
\end{aligned}
$$

Taking logarithms (deleting terms of order $O\left(r^{2} / x^{2}\right)$ ) we get (using $\nu=$ $r+3 / 2$ and $\left.\left(6 x-\nu^{2}\right) /\left(2\left(\nu^{2}+4 x\right)^{3 / 2}\right) \approx 1 /(16 \sqrt{x})\right)$

$$
\begin{aligned}
& \frac{1}{2} \log 32+r \log a+\sqrt{\nu^{2}+4 x}-\frac{1}{4} \log \left(\nu^{2}+4 x\right) \\
& -\nu \log \left(\nu+\sqrt{\nu^{2}+4 x}\right)+\frac{1}{16 \sqrt{x}}+\frac{r a^{2}}{24 x} \approx \sqrt{x}-\log x-\frac{1}{\sqrt{x}}+\frac{r a^{2}}{32 x} .
\end{aligned}
$$


C. Knessl has informed me that this equation agrees up to $O(1 / \sqrt{x})$ with Result 1 in [3]. Expand everything in sight in powers of $\nu / \sqrt{x}$ :

$$
\begin{aligned}
\sqrt{\nu^{2}+4 x} & =2 \sqrt{x}\left(1+\frac{\nu^{2}}{8 x}-\frac{\nu^{4}}{128 x^{2}}+\frac{\nu^{6}}{8192 x^{3}}-\ldots\right), \\
\log \left(\nu^{2}+4 x\right) & =\log 4+\log x+\frac{\nu^{2}}{4 x}-\frac{\nu^{4}}{32 x^{2}}+\frac{\nu^{6}}{192 x^{3}}-\ldots, \\
\log \left(\nu+\sqrt{\nu^{2}+4 x}\right) & =\int \frac{d \nu}{\sqrt{\nu^{2}+4 x}}=\frac{1}{2 \sqrt{x}} \int \frac{d \nu}{\sqrt{1+\nu^{2} /(4 x)}} \\
& =\frac{1}{2 \sqrt{x}} \int\left(1-\frac{\nu^{2}}{8 x}+\frac{3 \nu^{4}}{128 x^{2}}-\ldots\right) d \nu \\
& =\log 2+\frac{1}{2} \log x+\frac{\nu}{2 \sqrt{x}}-\frac{\nu^{3}}{48 x \sqrt{x}}+\frac{3}{1280} \frac{\nu^{5}}{x^{2} \sqrt{x}}-\ldots
\end{aligned}
$$

Substituting this we get

$$
\begin{aligned}
\frac{r}{2}(\log x-2 \log a+2 \log 2) \approx & \frac{1}{2} \log 2+\sqrt{x}-\frac{1}{4} \frac{\nu^{2}}{\sqrt{x}} \\
& +\frac{1}{192} \cdot \frac{\nu^{4}}{x \sqrt{x}}-\frac{43}{20480} \cdot \frac{\nu^{6}}{x^{2} \sqrt{x}}+\frac{17}{16} \cdot \frac{1}{\sqrt{x}} \\
& -\frac{\nu^{2}}{16 x}+\frac{1}{96} \cdot \frac{r a^{2}}{x}+\frac{\nu^{4}}{128 x^{2}}-\frac{\nu^{6}}{768 x^{3}} .
\end{aligned}
$$

Put

$$
x_{1}=\frac{4 x}{a^{2}}=\frac{24}{\pi^{2}}\left(n-\frac{r}{2}-\frac{1}{24}\right) .
$$

Then

$$
\begin{aligned}
r \log x_{1}= & \log 2+2 \sqrt{x}-\frac{1}{2} \cdot \frac{\nu^{2}}{\sqrt{x}}+\frac{1}{96} \cdot \frac{\nu^{4}}{x \sqrt{x}}-\frac{43}{10240} \cdot \frac{\nu^{6}}{x^{2} \sqrt{x}} \\
& +\frac{17}{8} \cdot \frac{1}{\sqrt{x}}-\frac{\nu^{2}}{8 x}+\frac{1}{48} \cdot \frac{r a^{2}}{x}+\frac{\nu^{4}}{64 x^{2}}-\frac{\nu^{6}}{384 x^{3}}+\ldots
\end{aligned}
$$

Using the fact that $\nu=r+3 / 2$ and solving for $r$ successively we get

$$
\begin{aligned}
(* *) r= & \frac{a \sqrt{x_{1}}}{\log x_{1}}\left\{1+\frac{1}{\log ^{2} x_{1}}+\frac{25}{12} \cdot \frac{1}{\log ^{4} x_{1}}-\frac{1803}{320} \cdot \frac{1}{\log ^{6} x_{1}}\right\} \\
& +\frac{\log 2}{\log x_{1}}-\frac{3}{\log ^{2} x_{1}}-\frac{1}{2} \cdot \frac{1}{\log ^{3} x_{1}}+\frac{10}{3} \cdot \frac{1}{\log ^{4} x_{1}}+\frac{5}{4} \cdot \frac{1}{\log ^{5} x_{1}} \\
& -\frac{2363}{320} \cdot \frac{1}{\log ^{6} x_{1}}+\ldots+\frac{2}{a} \cdot \frac{1}{\sqrt{x_{1}} \log x_{1}}+\frac{a}{12} \cdot \frac{1}{\sqrt{x_{1}} \log ^{2} x_{1}}+\ldots
\end{aligned}
$$

Now we want to invert the relation, i.e. solve for $x_{1}$ in terms of $r$. We begin 
by solving the equation

$$
r=\frac{a \sqrt{x_{1}}}{\log x_{1}}=\frac{a}{2} \cdot \frac{\sqrt{x_{1}}}{\log \sqrt{x_{1}}} .
$$

Make the substitutions

$$
r_{1}=\frac{2 r}{a}=\frac{12}{\pi^{2}} r, \quad t=\sqrt{x_{1}} .
$$

Hence $t=r_{1} \log t$ and we get

$$
\begin{aligned}
t & =r_{1} \log \left(r_{1} \log t\right)=r_{1}\left(\log r_{1}+\log \log t\right) \\
& =r_{1}\left(\log r_{1}+\log \log r_{1}+\log \log \log t\right)=\ldots
\end{aligned}
$$

We stop at

$$
t_{0}=r_{1}\left(\log r_{1}+\log \log r_{1}+\log \log \log r_{1}+\ldots\right)
$$

where we continue as long as the terms are positive. Then we take $t_{0}$ as an approximate solution of

$$
f(t)=t-r_{1} \log t=0
$$

and get a better solution $t_{1}$ by Newton-Raphson, i.e.

$$
t_{1}=t_{0}-\frac{f\left(t_{0}\right)}{f^{\prime}\left(t_{0}\right)}=t_{0}-\frac{t_{0}-r_{1} \log t_{0}}{1-\frac{r_{1}}{t_{0}}}=t_{0} \cdot \frac{\log t_{0}-1}{\frac{t_{0}}{r_{1}}-1} .
$$

Putting this into the original equation $(* *)$ we get

$$
x_{1} \approx \frac{t_{1}^{2}}{\left(1-\left(\frac{r_{1}}{2 t_{1}}\right)^{2}\right)^{2}} .
$$

Finally, a remark about the computation of $n_{0}(r)$. First, an approximate solution $x_{0}$ to the equation

$$
f(x)=L_{r+3 / 2}(x)-C L_{3 / 2}(x / 4)=0 \quad\left(\text { with } C=2^{-5 / 2}\left(12 / \pi^{2}\right)^{r}\right)
$$

is found using $(* * *)$. Then a better solution is obtained by using NewtonRaphson. We have

$$
f^{\prime}(x)=L_{r+5 / 2}(x)-\frac{1}{4} C L_{5 / 2}(x / 4)
$$

so

$$
x \approx x_{0}-\frac{L_{r+3 / 2}\left(x_{0}\right)-C L_{3 / 2}\left(x_{0} / 4\right)}{L_{r+5 / 2}\left(x_{0}\right)-\frac{1}{4} C L_{5 / 2}\left(x_{0} / 4\right)} .
$$

The procedure can be easily repeated.

Numerical examples. 1. Let $r=75$. Then the method in Theorem 2 gives $n_{0}=140235$. Iterating Newton-Raphson 5 times on $L_{r+3 / 2}(x)-$ 
$C L_{3 / 2}(x / 4)=0$ gives $n_{0}=140372.969$ suggesting that $n_{0}(75)=140372$, which is correct.

2 . Let $r=1000$. Then fitting in Theorem 1 gives $n_{0} \approx 53338036$. Then two iterations as above give $n_{0} \approx 53338056.74$ suggesting that $n_{0}(1000)=$ 53338056. The $L$-function was computed by adding 10000 terms. Using the more exact formula (with $L_{r+7 / 2}(x)$ etc.) did not change the result.

\section{References}

[1] M. Abramowitz and I. A. Stegun, Handbook of Mathematical Functions, Dover, New York 1965.

[2] G. Almkvist, Exact asymptotic formulas for the coefficients of nonmodular functions, J. Number Theory 38 (1991), 145-160.

[3] C. Knessl and J. B. Keller, Asymptotic behavior of high order differences of the partition function, Comm. Pure Appl. Math. 44 (1991), 1033-1045.

[4] A. M. Odlyzko, Differences of the partition function, Acta Arith. 49 (1988), 237254.

[5] H. Rademacher, Topics in Analytic Number Theory, Springer, Berlin 1973.

MATEMATISKA INSTITUTIONEN

LUNDS UNIVERSITET

22007 LUND, SWEDEN 